\title{
Recombinant expression and characterization of Solanum tuberosum aspartic protease
}

\author{
Lauren Agro, Brian Bryksa and Rickey Yada
}

\author{
This manuscript was prepared under the supervision of Professor Rickey Yada, \\ Department Food Science, Ontario Agricultural College
}

\begin{abstract}
Unique to aspartic proteinases from plants are the presence of approximately 100 amino acid regions, which are usually excised during activation of the zymogen. These sequences are termed 'Plant-Specific Inserts' and are implicated in membrane interactions of their parent enzymes, including vacuolar targeting and host defense. The need to further characterize the structure-function role(s) of plant-specific inserts stimulated the current study of the characterization of Solanum tuberosum AP (StAP). Recombinant expression of wild-type StAP resulted in the $54 \mathrm{kDa}$ protein being visualized by SDS-PAGE analysis with a protein yield of $0.03 \%$. A protein purification factor could not be established since activation of the protein at $\mathrm{pH}$ 2.2, 3.0, 3.7 and 5.5 was not achieved as evidenced by a lack of change in band patterns on SDS-PAGE as well as acidification and hemoglobin degradation assays. To potentially improve enzyme folding and activation ability, two mutants, (1) lacking the pre-signal sequence and (2) lacking both the signal sequence and the prosegment, were designed, sub-cloned, and expressed. Both products proved to be insoluble and inactive. New constructs were designed for the expression of StAP inclusion bodies for insoluble expression and subsequent re-folding of the protein. Additionally, Cys $\rightarrow$ Ala mutations for each PSI Cys residue were made to investigate the role(s) of plant-specific insert disulfide bonds in plant aspartic proteinase enzyme folding and structure. All PSI cysteine mutations (eight mutants) were successfully created using QuickChange Mutagenesis ${ }^{\mathrm{TM}}$.
\end{abstract}

A spartic proteases (APs) are a ubiquitous, heterologous class of proteinases that have been extensively studied. ${ }^{1}$ APs are characterized by having a prosegment that is cleaved when the zymogen is activated. Although APs display differences in activities and specificities, they share several common characteristics, including the fact that they contain two catalytic aspartate residues within two amino acid stretches that share a high degree of homology. ${ }^{1,2}$ The catalytic motifs are Asp-Thr-Gly and Asp-Ser-Gly in all plant APs belonging to the A1 family of proteases. ${ }^{3}$ APs also have catalytic activity at low $\mathrm{pH}$, a preference for hydrophobic amino acids, and are most often inhibited by pepstatin A. ${ }^{1,2}$ In addition, these proteases are characterized by a hydrophobic N-terminal signal sequence responsible for translocation into the ER, followed by a 40 amino acid prosegment that is cleaved upon zymogen activation. ${ }^{4}$

Currently, plant APs have not been studied as extensively as their mammalian counterparts. Known biological functions of plant APs are limited, and there is inadequate structure availability. ${ }^{1,2,4,5}$ Unique to plant APs, is the presence of a 100 amino acid sequence known as the plant-specific insert (PSI). ${ }^{2}$ The PSI is an independent subunit of five amphipathic $\alpha$-helices linked by three disulfide bridges inserted into the C-terminal domain. ${ }^{2}$ The PSI has been found to have high homology with mammalian saposins which are lysosomal, sphingolipid-activating proteins involved in membrane degradation. ${ }^{5}$ The PSI has been found to exhibit antimicrobial activity by inducing membrane leakage. ${ }^{5} \mathrm{~A}$ membrane-destabilizing domain of the PSI containing a high hydrophobic region possibly causes lipid disruption in vesicle membranes and resulting in pore formation and eventually cell death. ${ }^{5,6}$ It has also been suggested that the PSI is involved in vacuolar targeting of APs and is excised during activation. ${ }^{1,3}$

Only two crystal structures have been established for plant APs, including cardosin A and prophytepsin. ${ }^{3}$ Cardosin A, from Cynara cardunculus L. (Cardoon), is a milk-clotting AP used in cheese-making. The Cardosin precursor, Procardosin A, undergoes proteolytic processing as the cardoon flower matures. During this maturation, likely to occur in vacuoles, the PSI is removed before the removal of the prosegment. ${ }^{6}$

The present study seeks to further characterize the PSI in terms of its potential roles in membrane interactions and protein structure and function using Solanum tuberosum AP (StAP) as a model plant AP system. StAP has been shown to retain its PSI upon activation, ${ }^{5}$ thereby offering an opportunity to study PSI roles in an active AP conformation. The gene for StAP has previously been found to contain a coding region of $1494 \mathrm{bp}$ long including 497 amino acids out of a predicted $54 \mathrm{kDa}$ molecular mass. ${ }^{5}$ Neither StAP crystal structure nor the characterization of the StAP activation 
process have been studied to date -that is, both areas are poorly understood among plant APs. Guevara et al. determined that StAP possesses antimicrobial activity against P.infestans possibly by inducing membrane destabilization. ${ }^{4}$ Additionally, the disulfide bonds present in the PSI are proposed to function as a stabilizing factor for the AP. ${ }^{8}$ The goal of the present study was to express, purify, and partially characterize wild-type StAP proteolytic activity and activation in anticipation of a final crystal structure. Also, cysteine mutations were created in order to better understand the role of disulfide linkages within the PSI structure in StAP folding / structure, activation and activity.

\section{MAterials AND MethodS}

\section{Materials}

QuickChange Site-Directed Mutagenesis ${ }^{\mathrm{TM}}$ kit purchased from Stratagene (La Jolla, CA). Plasmid isolation kit purchased from Sigma (Oakville, ON). All primers were ordered from Sigma Genosys (Oakville, ON). Pfu Polymerase and PageRuler ${ }^{\mathrm{TM}}$ Pre-Stained Protein Ladder purchased from Fermentas Life Sciences (Burlington,ON). DC Protein Assay kit purchased from Bio-Rad Laboratories (Mississauga, ON). StAP sequence optimized for E.coli expression was purchased from Mr. Gene (Regensberg, Germany). pET vector system purchased from END Biosciences (San Diego, CA). All other reagents and supplies were purchased from Fisher Scientific (Ottawa, $\mathrm{ON})$.

\section{PSI Mutations}

The vector used to clone the optimized StAP sequence was a modified version of $\mathrm{pET} 32 \mathrm{~b}+$, containing a deletion of the STag and enterokinase coding sequences. Primers for $\mathrm{Cys} \rightarrow$ Ala mutagenesis were produced for each Cys residue of the PSI region of StAP. Cys $\rightarrow$ Ala mutations were located at C271A, C306A, C331A, C337A, C368A, C371A, C399A, and $\mathrm{C} 413 \mathrm{~A}$ corresponding to full-length (including signal sequence) StAP numbering. Primers were designed using PrimerX for QuickChange ${ }^{\mathrm{Tm}}$ site-directed mutagenesis (Table 2).

\section{Sub-cloning of truncated StAP forms}

StAP was produced without its pre-signal sequence (-P) in addition to StAP with pre-signal and prosegment deletion ($\mathrm{PP})$ in an attempt to increase solubility of the protein. Primers (Table 2) were ordered to clone -P StAP and -PP StAP into pET32(z)+ and a PCR reaction was completed using; 5uL of 10x Pfu $\mathrm{MgSO}_{4}$ reaction buffer, 0.5uL (250ng) of oligonucleotide fwd/rev primer, 1uL dNTP mix, 1uL Pfu DNA polymerase $(2.5 \mathrm{U} / \mathrm{uL}), 1 \mathrm{uL}$ dsDNA template (50ng/uL), and $41 \mathrm{uL}$ of $\mathrm{ddH}_{2} \mathrm{O}$. Cycle parameters consisted of denaturing at $95^{\circ} \mathrm{C}$ for 30 s followed by 18 cycles of $94^{\circ} \mathrm{C}$ (30s), $58^{\circ} \mathrm{C}(1 \mathrm{~min})$ and $72^{\circ} \mathrm{C}(15 \mathrm{~min})$.

\section{Construct verification}

Mutagenesis / ligation reactions were transformed into E. Coli TOP10F' competent cells using the Hanahan method. DNA sequencing was done by ACC Genomic Facility (Guelph, ON) to verify mutations. If correct, transformations were transformed into expression strain Rosetta Gami B DE3 pLysS (RG) using 1uL of stock plasmid DNA. Stock cultures were stored in $20 \%$ glycerol at $-80^{\circ} \mathrm{C}$.

\section{Expression of StAP}

One litre LB (Ap, Kn,Cm,Tet) was inoculated with $25 \mathrm{~mL}$ overnight culture and grown at $37^{\circ} \mathrm{C}$. Cultures were induced with $1 \mathrm{mM}$ isopropyl $\beta$-D-1-thiogalactopyranoside (IPTG) when $\mathrm{OD}_{600}$ reached between 0.5-0.8. Cultures were further incubated at $30^{\circ} \mathrm{C}$ for $3 \mathrm{~h}$. Cells were collected by centrifugation at $4,800 \mathrm{x}$ g at $4^{\circ} \mathrm{C}$ for $6 \mathrm{~min}$. Cell pellets were stored at $-20^{\circ} \mathrm{C}$. Additionally, -P StAP and -PP StAP strains were expressed at RT and $30^{\circ} \mathrm{C}$ to determine if expression of soluble fusion protein increased at lower expression temperature.

\section{Purification of StAP}

Frozen expression pellets were resuspended in $20 \mathrm{~mL} 20 \mathrm{mM}$ Tris- $\mathrm{HCl}$ pH 7.5 per L original expression culture. Samples were rotated for $60 \mathrm{~min}$ at RT to allow cell lysis (via pLysS). To reduce viscosity, $1 \mathrm{uL}$ Benzonase-Nuclease (EMD Biosciences, San Diego, CA) was added for every $40 \mathrm{~mL}$ of solution. The reaction mixture was continuously rotated at room temperature for $15 \mathrm{~min}$, after which the solution was then centrifuged at $4,800 \mathrm{x} \mathrm{g}$ at $4^{\circ} \mathrm{C}$ for $10 \mathrm{~min}$, after which the supernatant was re-centrifuged at $21,000 \mathrm{x} \mathrm{g}$ at $4^{\circ} \mathrm{C}$ for 30min.

Supernatant was loaded onto an Umac cobalt column (EMD Biosciences, San Diego, CA) using a Bio-Rad Econo Pump. Sample was washed with Buffer A $(20 \mathrm{mM}$ Tris/300mM NaCl pH 8.0) until the absorbance stabilized. Buffer B (20mM Tris/300mM NaCl/250mM Imidazole $\mathrm{pH}$ 8.0) was added at $4 \%, 10 \%$ and $100 \%$ step intervals to purify desired His-tagged fusion protein (StAP). Fractions containing StAP were dialyzed overnight in $20 \mathrm{mM}$ Tris- $\mathrm{HCl}$ pH 7.5 (total dilution factor 40,000-fold) in Fisher Brand dialysis tubing (12,000-14,000 MWCO) and protein concentration was assessed using DC protein assay (BioRad, Mississauga, ON). Thrombin $(0.02 \mathrm{mg} / \mathrm{mL}$ stock) was added to the dialysate at a protein mass ratio of 3000:1. Samples were left overnight on a rotator and aliquots were taken at 2 and 24 hours to view cleavage progress. Cobalt affinity HPLC was used to separate released StAP (no Histag) from fusion partner thioredoxin and uncleaved fusion protein (His-tag) by collecting flow-through. Samples were re-dialyzed as described above. Samples were loaded onto a $1 \mathrm{~mL}$ MonoQ column (GE Healthcare), washed with $20 \mathrm{mM}$ Tris- $\mathrm{HCl} \mathrm{pH} 7.5$, and eluted using a gradient $20 \mathrm{mM}$ Tris- $\mathrm{HCl}$, $2 \mathrm{M} \mathrm{NaCl}, \mathrm{pH} 7.5$. 


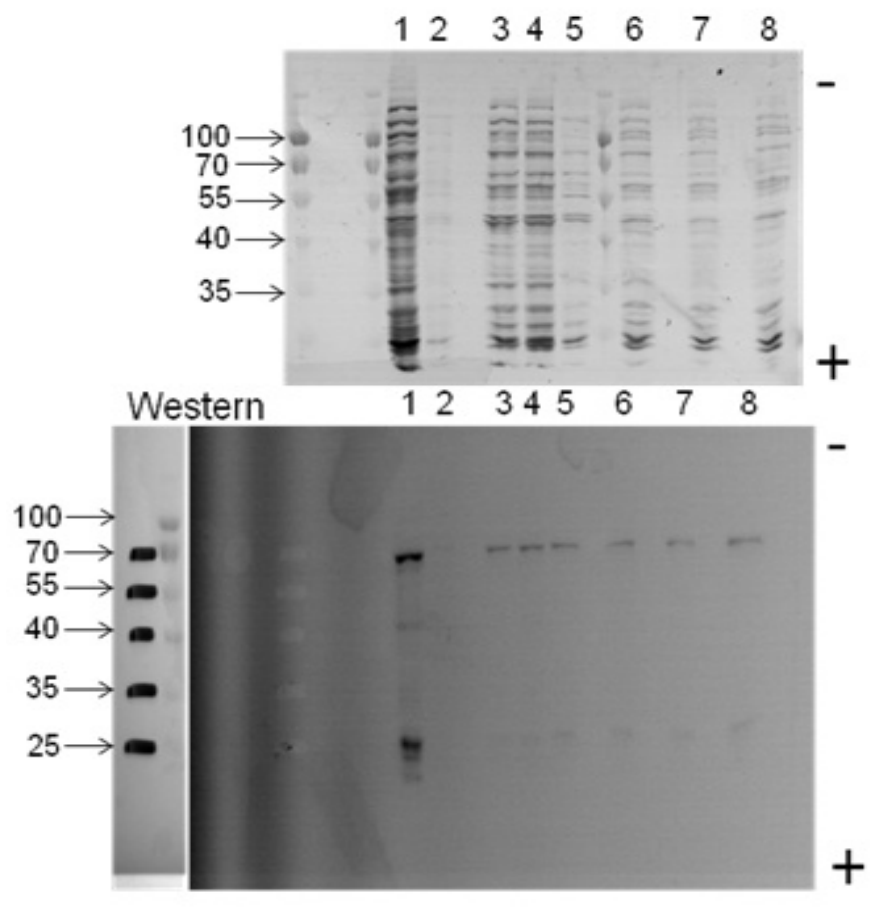

Figure 1: Bottom: Western blot using HisProbe ${ }^{\mathrm{TM}}$ to detect Histagged protein; Top: same blot stained with Coomassie G-250. Lane 1: Crude protein; Lane 2: Dilute crude protein; Lane 3-5: WP StAP post Co-HPLC; Lanes 6-8: a second isolate WP StAP Co-HPLC elution

\section{Activation full length, $-P$ and $-P P$ StAP}

Post-thrombin StAP was acidified by addition of the following buffers at the indicated concentrations to test for autoactivation similar to the method of Glathe et al. ${ }^{7} 50 \mathrm{mM}$ Glycine-HCl pH2.2, 50mM Na-citrate $\mathrm{pH} 3.0,50 \mathrm{mM} \mathrm{Na}-$ acetate $\mathrm{pH} 3.7$, and $50 \mathrm{mM} \mathrm{Na-acetate} \mathrm{pH}$ 5.5. Samples at various stages (time and $\mathrm{pH}$ ) of experimentation were analyzed using $12 \%$ and $18 \%$ SDS-PAGE to identify band shifting resulting from acidification. Aliquots were taken at 1 $\mathrm{h}, 5 \mathrm{~h}$, and $24 \mathrm{~h}$. Acidified StAP samples at the same $\mathrm{pH}$ values were also prepared in the presence of $1 \mathrm{mg} / \mathrm{mL}$ hemoglobin substrate to test for enzymatic activity

\section{Refolding of $-P$ StAP and $-P P$ StAP}

After $24 \mathrm{~h}$ dialysis in $20 \mathrm{mM}$ Tris-HCl pH 7.5, -P StAP fusion protein solution samples appeared cloudy. 50uL of sample was centrifuged for $10 \mathrm{~min} 14,000 \mathrm{xg}$ to visualize insoluble protein on SDS-PAGE. The supernatant was collected and the pellet washed twice with $20 \mathrm{mM}$ Tris- $\mathrm{HCl} \mathrm{pH} 7.5$. Insoluble $-\mathrm{P}$ StAP pellets were refolded using $8 \mathrm{M}$ urea containing $100 \mathrm{mM} \beta$-mercaptoethanol as done with Cardosin A by Castanheira et al. ${ }^{8}$

\section{RESULTS AND DISCUSSION}

First attempts at mutagenesis were successful for C271A, C306A, C331A, C368A, and C413A StAP, however, C337A, C371A and C399A StAP yielded wild-type. A second attempt at mutagenesis yielded successful C337A and C371A mutants. After reviewing primers for C399A primers, secondary structures that could interfere with mutagenesis reaction were identified and thus a new primer was designed and used yieding C399A StAP. Wild-type and mutants were transformed using E. coli strain RG. Wild-type, and truncated, StAP were expressed as described in the Materials and Methods section.

When purifying StAP using cobalt affinity HPLC, it was found that washing using $4 \%, 10 \%$ buffer $\mathrm{B}$, followed by eluting at $100 \%$ buffer B, yielded improved purity relative to washing using only Buffer A (data not shown). Only 100\% B eluate contained target fusion protein (Figure 1). The total soluble target fusion protein recovery was determined to be $0.03 \%$ of the total soluble crude protein (Table 1). Fusion protein samples were dialyzed prior to thrombin cleavage to remove imidazole in order to allow for a second Cobalt affinity HPLC step, allowing for the retention of His-tagged

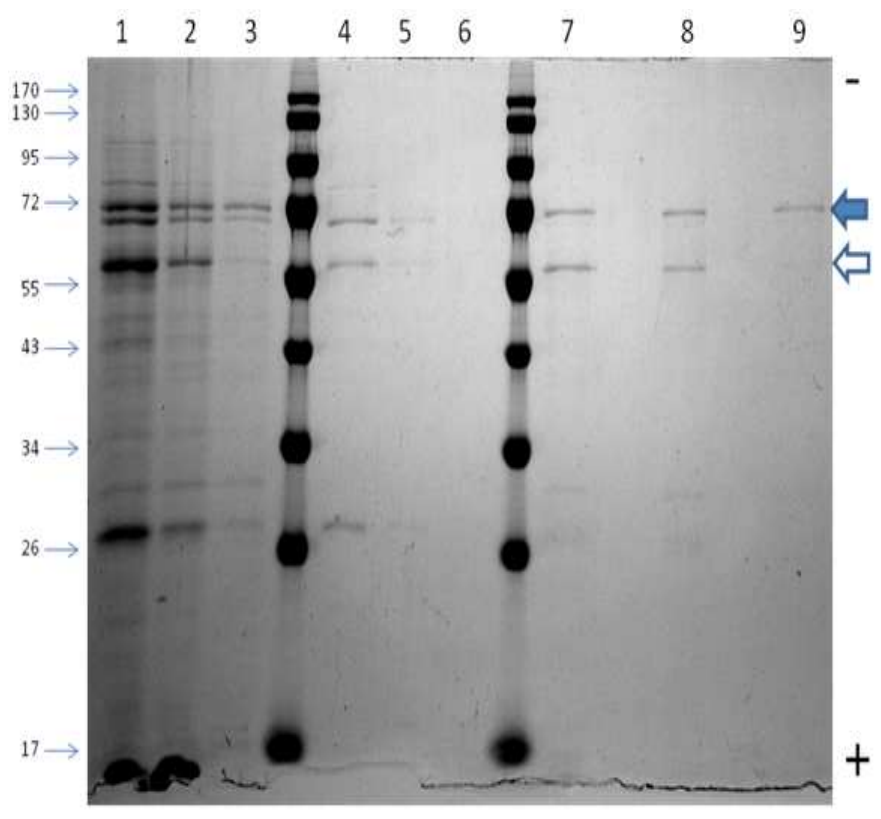

Figure 2: 12\% SDS-PAGE. WT StAP isolation from fusion tag; Lanes 1-3: Co HPCL eluate fractions post-thrombin; Lanes 4-6: Flow-through Co HPCL fractions post-thrombin; Lanes 7-9; Co HPCL eluate fractions post-thrombin. The solid arrow indicates uncut Trx-StAP fusion protein MW; the white arrow indicates StAP MW. 


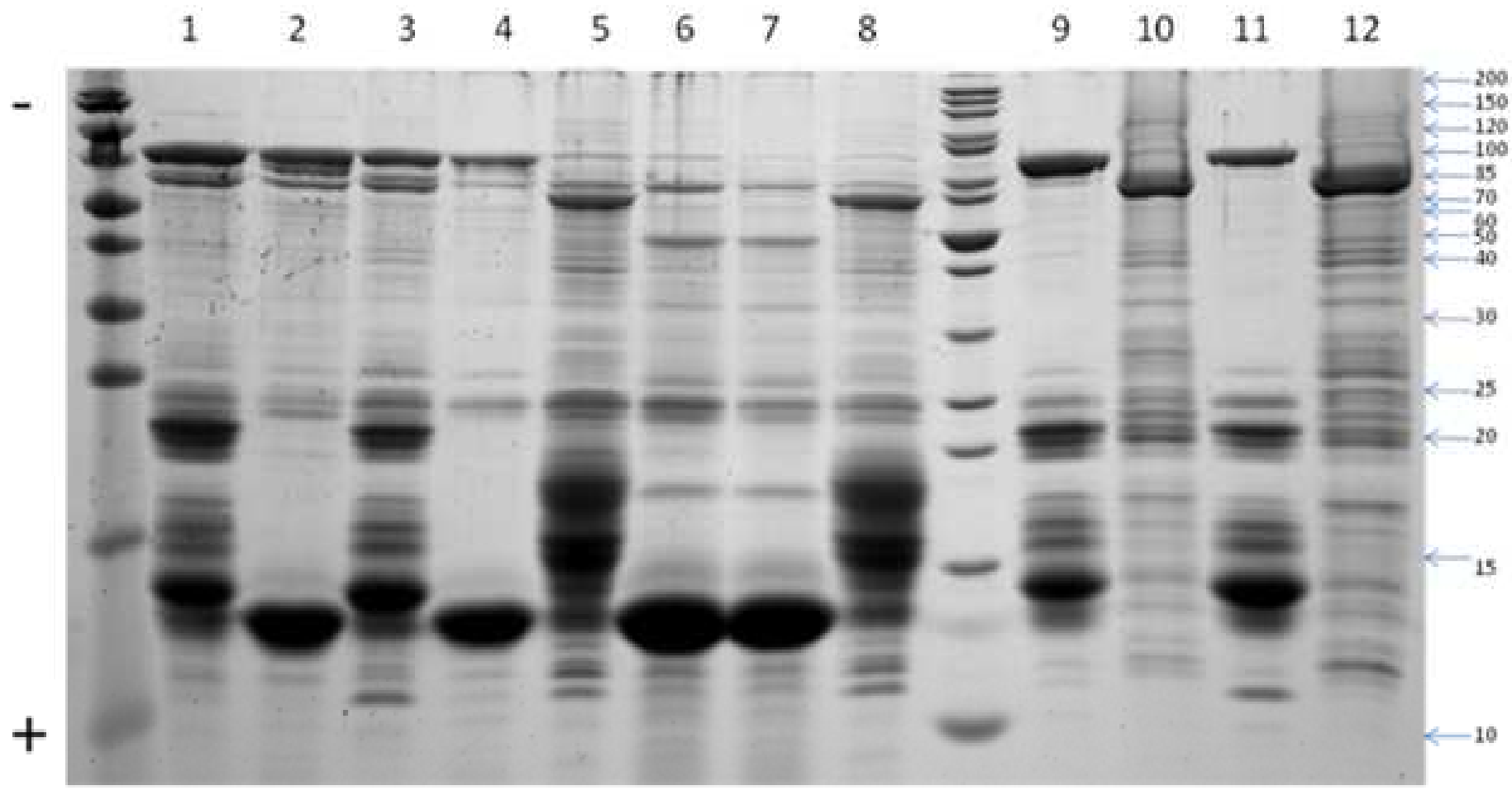

Figure 3: 18\% SDS_PAGE; Lanes 1 and 3: -P StAP post-Co HPLC; Lanes 2 and 4: Post thrombin cleavage -P; Lanes 5 and 8: -PP StAP post -Co HPLC; Lanes 6 and 7: Post-thrombin cleavage -PP; Lanes 9 and 11: Soluble fraction -P STAP; Lanes 10 and 12: insoluble fraction -P StAP

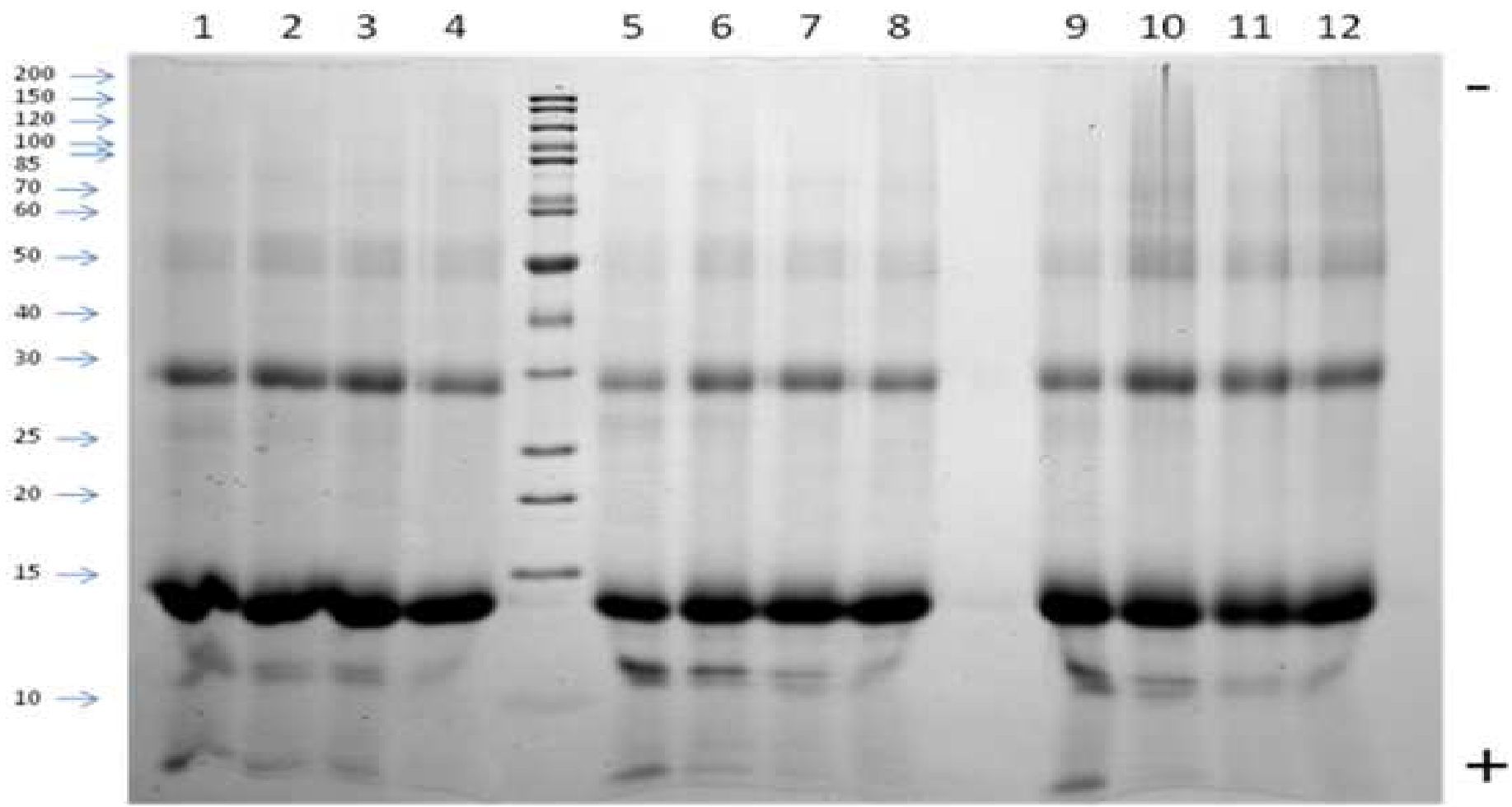

Figure 4: 18\% SDS-PAGE; Hemoglobin degradatopm assays after $17 \mathrm{~h}$ incubation at $37^{\circ} \mathrm{C}$; lanes 1-4: Controls for hemoglobin acidification tests at pH 2.2, 3.0, 3.7 and 5.5 respectively; Lanse 5-8: -P StAP at the same pH values, respectively; Lanes 9-12: PPStAP at above pH values. 


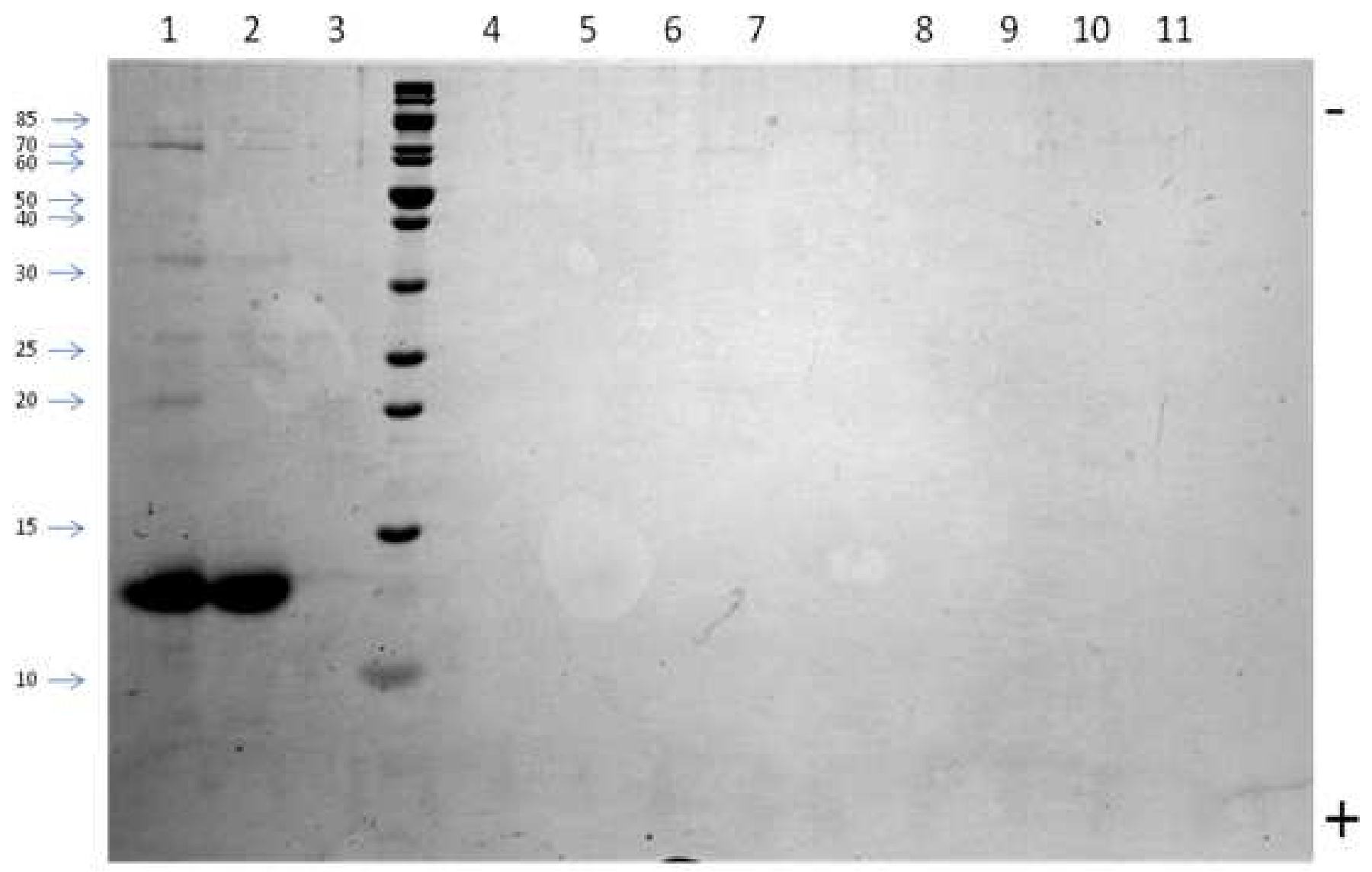

Figure 5: 18\% SDS_PAGE. Lane1: -P StAP post-thrombin soluble fraction; Lane 2: Post-thrombin -P StAP soluble fraction; Lane 3: -'refolding' -P StAP; Lanes 4-7 and 8-11 represent $2 \mathrm{~h}$ and $18 \mathrm{~h}$ acidification of 'refolded' -P StAP, respectively.

thioredoxin, while target StAP was allowed to flow through (Figure 2). Following dialysis and further HPLC of the cleaved wildtype StAP, a new band on SDS-PAGE appeared corresponding to the molecular weight of wild-type StAP $(54.3 \mathrm{kDa})$. An additional band appeared just below $70 \mathrm{kDa}$ corresponding to the uncleaved fusion protein $(68.2 \mathrm{kDa})$. Therefore, thrombin cleavage was incomplete. Thioredoxin tag containing the His-tag and thrombin cleavage site was visualized just below the $17 \mathrm{kDa}$ standard. After attempting activation of the wild-type StAP at pH 2.2, 3.0, 3.7 and 5.5 similar to the method of Glathe et al. ${ }^{7}$ no band shifting was observed, i.e., a band shift indicates cleavage of the zymogen to produce mature enzyme (data not shown). Furthermore, no hemoglobin proteolysis was apparent under the above $\mathrm{pH}$ condition upon incubation for up to $48 \mathrm{~h}$ at $37^{\circ} \mathrm{C}$.

In an attempt to improve StAP folding and activation ability, two truncated forms were created, -P and -PP, eliminating the pre- signal sequence (Gly25 N-terminus) and both the pre- signal sequence and the prosegment (Asp68 Nterminus), respectively. The signal sequence in StAP consists of residues $1-24 .^{5}$ AP signal sequences are removed prior to activation, ${ }^{9}$ thus it was hypothesized that $-\mathrm{P}$ StAP expression may result in an enzyme capable of activation in vitro. -PP StAP was designed to test if StAP requires prosegment for correct folding as active form StAP has been shown to be stable at non-acidic $\mathrm{pH}$ values. ${ }^{10}$ Expression Vlevels of both StAP truncated forms at room temperature versus $30^{\circ} \mathrm{C}$ were compared to determine if lowered induction temperature improved target protein expression. $-\mathrm{P}$ StAP RT and $30^{\circ} \mathrm{C}$ contained $1.8 \mathrm{mg}$ and $2.9 \mathrm{mg}$ total protein, respectively. -PP StAP RT and $30^{\circ} \mathrm{C}$ samples contained $0.50 \mathrm{mg}$ and $3.1 \mathrm{mg}$ total protein, respectively (Table 1). Thus, RT induction yielded lower total protein content, however, SDS-PAGE revealed that RT induction yielded increased purity as fewer contaminant bands were visualized (data not shown).

Fusion protein masses for -P-Trx and -PP-Trx were 66 $\mathrm{kDa}$ and $61 \mathrm{kDa}$, respectively. Thrombin treatment of the truncated StAP forms (-P and -PP) produced the expected new bands at approximately $52 \mathrm{kDa}$ for $-\mathrm{P}$ StAP and $47 \mathrm{kDa}$ for PP StAP (Figure 3). Upon dialysis of -P StAP with $20 \mathrm{mM}$ Tris- $\mathrm{HCl} \mathrm{pH} 7.5$ and thrombin cleavage, the sample 
Table 1: Protein purification table for WT Trx-StAP partial purification. Protein purification factor could not be calculated in the absence of specific activity measurements. -P and -PP samples are also shown for comparison to WT StAP

\begin{tabular}{|l|l|l|l|l|}
\hline & Volume $\mathbf{( m L})$ & Protein $\mathbf{( m g} / \mathbf{m} \mathbf{L})$ & Total Protein $(\mathbf{m g})$ & Recovery $(\%)$ \\
\hline Crude Trx-StAP & 150 & 3.0 & 450 & 100 \\
\hline Co Elution WT & 1.5 & 0.83 & 1.2 & $0.027 \%$ \\
\hline Co Elution -P StAP RT & 3.0 & 0.98 & 2.9 & - \\
\hline Co Elution -P StAP 30 & 3.0 & 0.61 & 1.8 & - \\
\hline Co Elution -PP StAP RT & 2.5 & 0.20 & 0.50 & - \\
\hline Co Elution -PP StAP 30 & 3.5 & 0.89 & 3.1 & - \\
\hline
\end{tabular}

Table 2: Designed primers using PrimerX for QuickChange ${ }^{\mathrm{Tm}}$ site-directed mutagenesis. Primers were ordered to clone $-\mathrm{P}$ StAP and -PP StAP into pET32(z)+

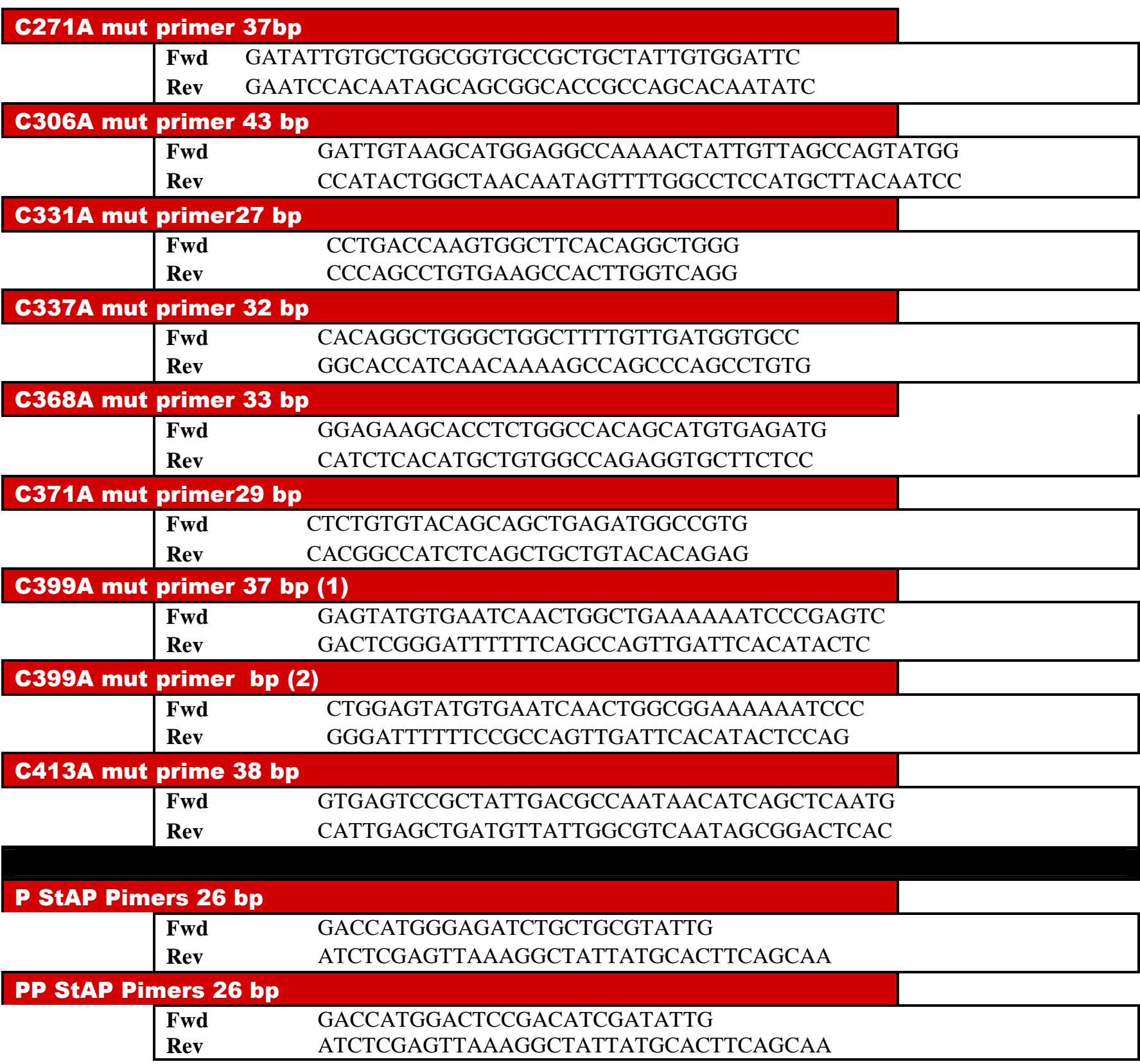


appeared 'cloudy' suggesting loss of solubility of StAP upon release from the highly soluble fusion partner thioredoxin (Trx). Centrifugation yielded an insoluble protein pellet. The pellet was solubilized in SDS-PAGE sample buffer and compared to the sample's soluble fraction on SDS-PAGE. Results indicated that the majority of the desired -P StAP became insoluble upon thrombin cleavage and dialysis (Figure 3). Apparently -P StAP solubility was dependent on the Trx fusion tag.

Activation of -P StAP and -PP StAP was attempted at $\mathrm{pH}$ 2.2, 3.0, 3.7 and 5.5 as used for full-length StAP. No clear band shift was visible suggesting that autoactivation of $-\mathrm{P}$ StAP protein had no occurred (data not shown). -PP StAP would not be expected to change masses as no prosegment was present by design. Additionally, proteolytic activity testing by hemoglobin degradation assays for $-\mathrm{P}$ and $-\mathrm{PP}$ StAP resulted in no detectable change in band patterns on SDS-PAGE as compared to the controls indicating no activity (Figure 4).

After dialysis, -P StAP was insoluble as noted above. Pellets collected from -P StAP centrifugation, as well as postthrombin (soluble) -PP StAP sample were subjected to the denaturing conditions of $8 \mathrm{M}$ urea and $100 \mathrm{mM} \quad \beta$ mercaptoethanol, followed by washing 3 times using $60 \mathrm{~mL}$ $20 \mathrm{mM}$ Tris- $\mathrm{HCl} \mathrm{pH} 7.5$ via dia-filtration (27,000-fold total dilution factor). Activation was again attempted at four different $\mathrm{pH}$ values and subsequent hemoglobin degradation assays were completed. No bands were visible on a SDSPAGE gel (Fiomg. 5) indicating that the protein reprecipitated upon washing with $20 \mathrm{mM}$ Tris- $\mathrm{HCl}$ pH 7.5 during refolding.

\section{FUTURE EXPERIMENTATION}

Future studies will attempt to obtain pure, folded StAP via expression in E.coli BL21 from refolded protein inclusion bodies, as refolding of protein inclusion bodies has been successfully used for Cardosin A. ${ }^{8}$ Expression of soluble StAP could be attempted using a Baculovirus-infected insect cell expression system. Such expression systems sometimes produce high levels of soluble target protein while allowing for post-translational modifications. Such modifications may help with the folding of StAP.

\section{REFERENCES}

1. Payie, K., Tanaka, T., Gal, S., and Yada, R. (2003) Construction, expression and characterization of a chimaeric mammalian-plat aspartic proteinase. Biochem. J. 372, 671678.

2. Mazorra-Manzano, M. and Yada, R. (2008) Expression and characterization of the recombinant aspartic proteinase A1 from Arabidopsis thaliana. Phytochemistry. 69, 24392448.

3. Simoes, I. and Faro, C. (2004) Structure and function of plant aspartic proteinases. Eur. J. Biochem. 271, 2067-2075.

4. Mendieta, J.R., Pagano, M.R., Munoz, F.F., Daleo, G.R. and Guevara, M.G. (2006) Antimicrobrial activity of potato aspartic proteases (StAPs) involves membrane permeabilization. Microbiology. 152, 2039-2047.

5. Guevara, M.G., Almeida, C., Mendieta, J.R., Faro, C.J., Verissimo, P., Pires, E.V. and Daleo, G.R. (2005) Molecular cloning of a potato leaf cDNA encoding an aspartic protease (StAsp) and its expression after P.infestans infection. Plant Physiology and Biochemistry. 43, 882-889.

6. Pereira, C., da Costa, D.S., Pereira, S., de Moura Nogueira, F., Albuquerque, P.M., Teixeira, J., Faro, C. and Pissarra, J. (2008) Cardosins in postembryonic development of cardoon: towards an elucidation of the biological function of plant aspartic proteinases. Protoplasma. 232, 203-213.

7. Glathe, S. Kervinen, J., Nimtz, M., Li, G.H., Tobin, G.J., Copeland, T.D., Ashford, D.A., Wlodawer, A. and Costa, J. (1998) Transport and activation of the vacuolor aspartic proteinase phytepsin in barley (Hordeum vulgare L.) J. Biol. Chem. 273, 31230-31236.

8. Castanheira, P. , Samyn, B., Sergeant, K., Clemente, J.C., Dunn, B.M., Pires, E., Van Beeumen, J. and Faro, C. (2005) Activation, proteolytic processing, and peptide specificity of recombinant cardosin A. J. Biol. Chem. 280, 13047-13054.

9. Runeberg-Roos, P., Kervinen, J., Kovaleva, V., Raikhel, N.V. and Gal, S. (1994) The aspartic proteinase of barley is a vacuolar enzyme that processes probarley lectin in vitro. Plant Physiol. 105, 321-329.

10. Guevara, M.G., Daleo, G.R. and Oliva, C.R. (2001) Purification and characterization of an aspartic protease from potato leaves. Physiologia plantarum 112, 321-326. 